\title{
¿Los riesgos de suicidio y eutanasia están cubiertos en el contrato de seguro?
}

\section{Are suicide and euthanasia risks covered in the insurance contract?}

Rolando Torres Gamero* https://orcid.org/0000-0001-7312-1717

http://dx.doi.org/10.21503/lex.v19i28.2319

* Abogado por la UNMSM desde el año 1986, ejerció la docencia universitaria en las Facultades de Derecho de las Universidades Tecnológica del Perú, Garcilaso de la Vega y Alas Peruanas, presidió la Comisión de Estudio de Seguros del Ilustre Colegio de Abogados de Lima (2013-2016) la cual integró desde el año 1999 hasta 2017, Jefe del Departamento Legal de Royal \& Sunalliance Seguros Fénix donde laboró desde el año 1985 hasta el 2002; expositor en el Diplomado de Seguros en IPAE desde 1999 hasta 2009 y en la actualidad profesor de las Escuelas de Seguros de la Asociación Peruana de Empresas Corredoras de Seguros (APECOSE) desde 1999 y del Instituto Peruano de Seguros (IPS) desde 2009. Perú.

Correo electrónico: rtorresgamero@gmail.com

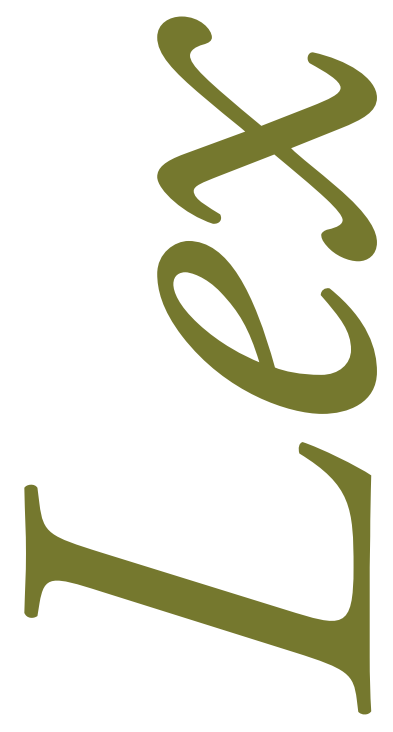
(C) (C) Los autores. Artículo publicado por la Revista Lex de la Facultad de Derecho y Ciencias Políticas de la Universidad Alas Comercial-Compartir Igual 4.0 Internacional.(http://creativecommons.org/licenses/by-nc-sa/4.0/), que permite el uso no comercial, distribución y reproducción en cualquier medio, siempre que la obra original sea debidamente citada. 


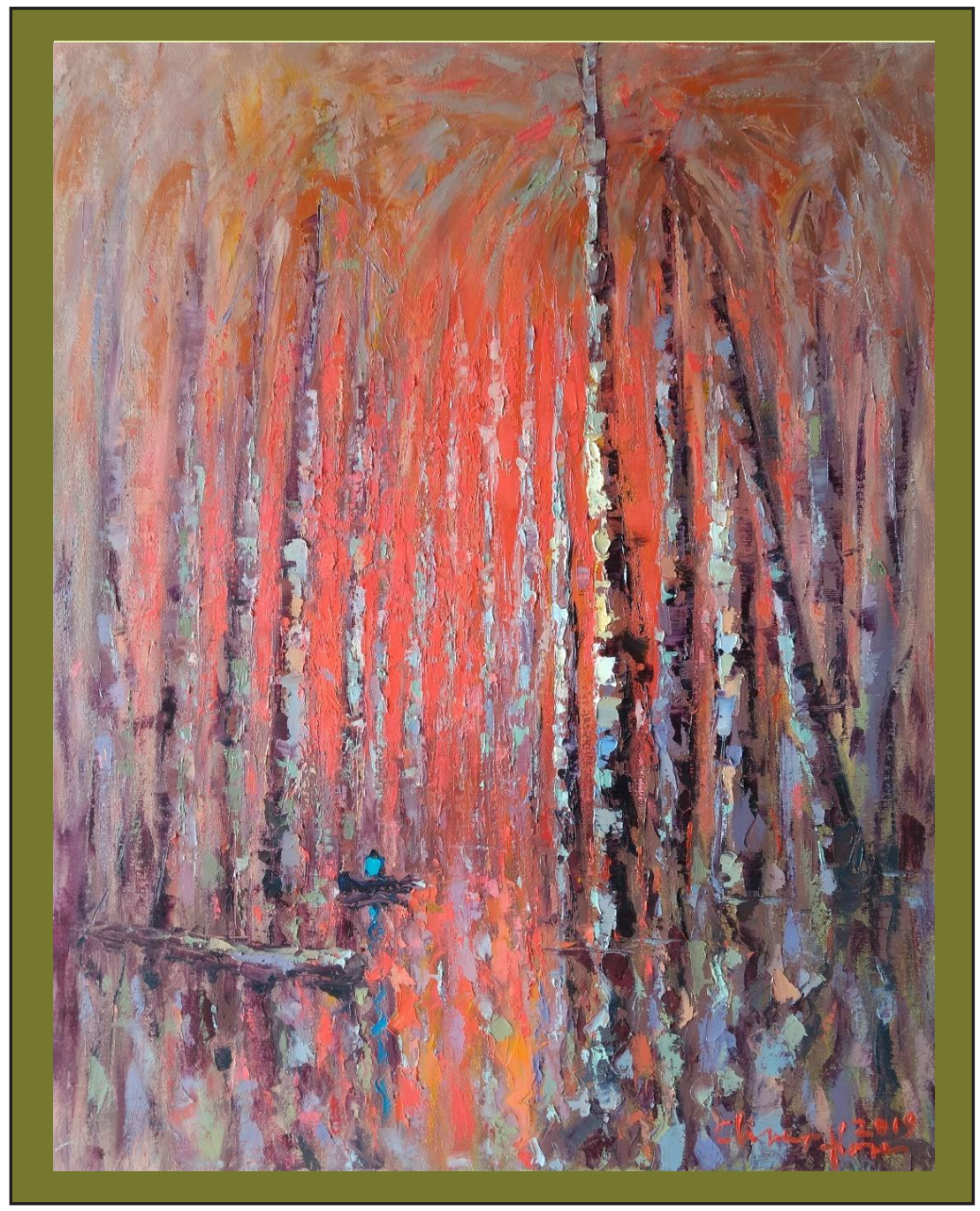

Luna llena en el aguajal. Óleo sobre lienzo, 50 X $61 \mathrm{~cm}$.

Cliver Flores Lanza (Iquitos, Loreto, Perú , 1965)

Correo electrónico: floreslanza@yahoo.com www.cliverpintoramazonico.blogspot.com 


\section{RESUMEN}

El presente trabajo busca establecer cómo las figuras del suicidio y la eutanasia como riesgos dentro del contrato de seguro están o no cubiertos generalmente en los seguros de vida y afines, si bien es cierto la naturaleza de esta cobertura es indemnizar a los beneficiarios de aquellos asegurados que pierden la vida por enfermedad (en forma natural) o por accidente (debidamente probado); el suicidio y la eutanasia vienen a ser unas formas de fallecimiento cuya muerte no está originariamente contemplada pero si de manera posterior, como en el caso del suicidio, como lo prevé nuestra Ley del Contrato de Seguro en el Perú promulgada en noviembre de 2012.

Palabras clave: suicidio, eutanasia, vida, seguro, cobertura.

\section{ABSTRACT}

This work seeks to establish how the figures of suicide and euthanasia as risks within the insurance contract are or are not generally covered in life and related insurance, although it is true the nature of this coverage is to compensate the beneficiaries of those insured who lose their lives due to illness (naturally) or by accident (duly proven); Suicide and euthanasia are forms of death whose death is not originally contemplated but later, as in the case of suicide, as provided by our Insurance Contract Law in Peru promulgated in november 2012.

Key words: suicide, euthanasia, life, insurance, coverage. 
"El único problema filosófico verdaderamente serio es el Suicidio. Juzgar si la vida es o no digna de vivir es la respuesta fundamental a la suma de preguntas filosóficas."

ALBERT CAMUS

\section{INTRODUCCIÓN}

Dos acontecimientos han sorprendido a nuestro país teniendo estos como base las dos figuras materia de este estudio: el suicidio y la eutanasia.

1. El 17 de abril de 2019, ante la presencia de un Fiscal y una dotación policial que contaban con una orden de detención preliminar contra su persona, el Dr. Alan García Pérez, dos veces Presidente Constitucional del Perú (1985-1990 y 2006-2011) ingresó a su dormitorio y tomó la fatal decisión de suicidarse.

Este evento trágico llevó a diversas especulaciones sobre las circunstancias que llevaron a dicho desenlace y, no faltó (independientemente del análisis político, policial, etc.) quien relacionara el mismo con el de un seguro de vida indicándose que dicho accionar no sería indemnizado por falta de cobertura para dicho riesgo.

2. Desde el año 2019 doña Ana Milagros Estrada Ugarte, psicóloga de profesión, quien padece de polimiositis, una enfermedad incurable, degenerativa y progresiva, en etapa avanzada, solicitó al Estado Peruano se le permita acceder a una muerte asistida; sólo recibió el apoyo de la Defensoría del Pueblo con quienes interpone demanda de Amparo contra Norma Legal a fin de que se declare inaplicable el artículo $112^{\circ}$ del Código Penal Peruano (Decreto Legislativo $N^{\circ}$ 635) que tipifica el delito de homicidio piadoso, con la finalidad de que ella pueda elegir, sin que terceros sean procesados penalmente, el momento en el cual las emplazadas (Seguro Social de Salud, Ministerio de Salud, Ministerio de Justicia y Derechos Humanos) deberán procurarle un procedimiento médico de eutanasia, para el cese de su vida cuando, debido a los intolerables dolores de la enfermedad que padece y a las condiciones de deterioro de su salud que derivan de esta, prolongar su existencia sea incompatible con su dignidad; dicha Acción de Amparo fue interpuesta el 31 de Enero de 2020. 
Con fecha 22 de febrero de 2021, el Juez del Décimo Primer Juzgado Constitucional de Lima emite Sentencia declarando FUNDADA EN PARTE LA DEMANDA interpuesta por la Defensoría del Pueblo en beneficio de dońa Ana Estrada Ugarte contra los emplazados al considerarse afectados los derechos a la dignidad, autonomía, libre desarrollo de su personalidad y de la amenaza de no sufrir tratos crueles e inhumanos. En consecuencia, consentida que sea la sentencia; se dispone que se inaplique el artículo $112^{\circ}$ del Código Penal vigente (delito de homicidio piadoso), para el caso de dońa Ana Estrada Ugarte y además se ordene al Ministerio de Salud y a EsSalud respetar la decisión de doña Ana Estrada Ugarte, de poner fin a su vida a través del procedimiento técnico de la eutanasia. Definitivamente, una Resolución Judicial sin precedentes en el Perú ante un pedido insólito por lo menos en una sociedad conservadora aún como la nuestra. Cabe resaltar que las entidades emplazadas no interpusieron recurso impugnatorio alguno contra dicha sentencia quedando en consecuencia firme, adquiriendo en consecuencia, autoridad de cosa juzgada.

No es nuestra intención analizar las razones que llevaron a que, en primer lugar, el citado personaje político haya decidido dar fin a su vida suicidándose y en segundo término, el de dońa Ana Estrada Ugarte el de recurrir vía la Defensoría del Pueblo a ser autorizada judicialmente a dar fin a su vida vía la eutanasia, sino el de analizar, conforme al título del presente trabajo, si ambos riesgos cuentan con cobertura en el contrato de seguro, y en especial, en el seguro de vida para lo cual revisaremos tanto la legislación nacional como comparada.

\section{EL SUICIDIO}

El suicidio es la acción personal, voluntaria, de quitarse la propia vida.

Es el suicidio uno de los problemas más graves de salud de los últimos años como lo califica un estudio realizado por Avalia-t, Agencia de Conocimiento en Salud de España ${ }^{1}$ remontándose a datos del año 2008 donde el suicidio en dicho país fue la primera causa de muerte violenta, superando a los accidentes de tráfico.

La Organización Panamericana de la Salud, filial Perú, OPSPerú, reporta un Informe de la Organización Mundial de la Salud desde Ginebra el 09 de septiembre de 2019 donde señalan que "a pesar del progreso en las estrategias de prevención, una persona todavía muere cada 40 segundos por suicidio" 2 .

El suicidio continúa siendo un problema de salud pública de gran relevancia en las Américas, donde constituye la 21.ava principal causa de muerte. Cada año más de 81,000 personas se quitan la vida en

1. Avalia-t, Agencia de Conocimiento en Salud, Galicia, España, documento elaborado por el Grupo de Trabajo de la Guía Práctica de Prevención y Tratamiento de la Conducta Suicida. (Madrid: Ministerio de Sanidad, Política Social e Igualdad. Axencia de Avaliación de Tecnoloxias Sanitarias de Galicia (avalia-t) 2010). Guías de Práctica Clínica en el SNS: avalia-t No 2010/02.

2. OPS, Boletín Setiembre 2019, mediateam@paho.org 
la región, el 56\% de ellas en Estados Unidos y Canadá. En las últimas décadas y en particular desde el año 2000, se han desarrollado varias estrategias de prevención del suicidio, indica el citado informe.

Este año 2021, la OPS, en un reciente Boletín Informativo en el marco del Día Mundial para la Prevención del Suicidio que tiene lugar el 10 de Septiembre, advirtió que la pandemia por COVID-19 ha exacerbado los factores de riesgo asociados a las conductas suicidas y llama a priorizar su prevención. ${ }^{3}$

$\mathrm{Al}$ respecto la OPS establece que la pandemia ha amplificado los factores de riesgo asociados al suicidio, como la pérdida de empleo o económica, los traumas o abusos, los trastornos mentales y las barreras de acceso a la atención de salud. Un año después del inicio de la pandemia, alrededor del 50\% de las personas que participaron en una encuesta del Foro Económico Mundial en Chile, Brasil, Perú y Canadá declararon que su salud mental había empeorado.

Refiriéndonos estrictamente a nuestro país, encontramos un estudio publicado el 2016 sobre la materia denominado "Evolución y Diferencias Regionales de la Mortalidad por Suicidios en el Perú, 2004-2013" elaborado por un grupo de investigadores ${ }^{4}$ que dentro de sus hallazgos encontraron que un total de 3,162 suicidios fueron registrados en la base de datos de defunciones del MINSA en el período 2004-2013. Del total de eventos reportados, un 67,2\% fueron hombres; el grupo etario de 20 a 29 años fue el grupo de mayor incidencia con $28,7 \%$ del total de casos, y el 49,2\% de eventos ocurrió por envenenamiento. El promedio de edad de los suicidas fue de 34,5 años.

El presente año 2021, mediante Nota de Prensa del pasado 1 de Mayo, el Ministerio de Salud (MINSA) advirtió que la tasa de suicidio está en aumento en el Perú, tanto así que en los primeros cuatro meses de este año, más de 200 personas decidieron acabar con sus vidas lo que podría significar que para finales de diciembre la cifra de suicidas supere los 614 que se registró en todo el 2020, debido principalmente al impacto de la pandemia por la COVID-19 que ha generado ansiedad y estrés crónico en la población. La médica psiquiatra del Hospital Víctor Larco Herrera, Julissa Castro, destacó que la carencia del acompańamiento social de la familia y amistades son factores importantes para esta tendencia. ${ }^{5}$

Lamentablemente, el pasado 11 de Setiembre en nota aparecida en el diario Perú 21 se publican declaraciones del Director de Salud Mental del MINSA, Dr. Yuri Cutipe, quien señaló que en los primeros seis meses de este 2021, un total de 337 personas se han quitado la vida, 167 en el primer trimestre y 170 en los tres meses restantes.

\footnotetext{
3. OPS, Boletín setiembre 2021, mediateam@paho.org

4. A. Hernández-Vásquez, D. Azañedo, J.Rubilar-González, B. Huarez, L. Grendas, "Evolución y diferencias regionales de la mortalidad por suicidios en el Perú, 2004-2013”, Rev. Perú Med. Exp. Salud Pública, 33 (4) (2016): 33 (4) 751-7, http://doi.org/10.17843/rpmesp.2016.334.2562

5. Gob.pe, Plataforma digital única del Estado Peruano, MINSA, Noticias
} 
Menciono estas dos notas de prensa como una peligrosa proyección en incremento de lo que los propios especialistas han podido prever sea a fin de año el penoso resultado estadístico de suicidios en nuestro país, ahora elevada más aún por la pandemia del Covid-19.

Émile Durkheim, sociólogo y filósofo francés considerado uno de los padres fundadores de la sociología se pronunció sobre esta figura indicando que "se llama suicidio todo caso de muerte que resulte directa o indirectamente de un acto positivo o negativo, ejecutado por la propia víctima, a sabiendas de que habría de producir éste resultado. La tentativa es ese mismo acto, pero interrumpido antes de que sobrevenga la muerte" ${ }^{6}$

Siguiendo a Durkheim establece tres categorías de suicidios ${ }^{7}$ :

a) Suicidio egoísta: Es aquel que resulta de la alienación del individuo respecto de su medio social. Este tipo es común allí donde factores culturales, como en el protestantismo, subrayan el individualismo y el esfuerzo concentrado en el yo, factor determinante en esta modalidad de autosacrificio.

b) Suicidio altruista: Es el que se encuentra en sociedades rígidamente estructuradas que ponen por encima del individuo un código de deberes de sentido grupal y hacen del sacrificio por el grupo una exigencia moral.

c) Suicidio anómico: Es el que se da cuando existe una falla o dislocación de los valores sociales, que lleva a una desorientación individual y a un sentimiento de falta de significación de la vida.

Finalmente, el psicólogo Andrés Felipe Palacio, estudioso de la obra de Durkheim, señala refiriéndose al sociólogo y filósofo francés que, sobre el tema del suicidio llega a una conclusión contundente: existirían sociedades suicidógenas, o sea, sociedades donde el suicidio es una tendencia, como podría serlo la pobreza, ya que es una de las consecuencias directas de lo que sucede en el discurso de dichas sociedades, sobre todo con respecto a la administración de sus reglas. ${ }^{8}$

Considero importante destacar estos estudios sobre el tema del suicidio sin pretender profundizar en los mismos por no ser el objetivo del presente trabajo, lo que sí es intención tratar de comprender porque luego en el ámbito del seguro se ha llegado prácticamente a aceptar dar cobertura a este riesgo en los seguros de vida con limitaciones en el tiempo de contratación como lo veremos más adelante tanto en nuestra legislación de la materia como en el derecho comparado.

6. Durkheim, Émile (1858, 1917), El Suicidio, Estudios de Sociología (versión directa del francés de Lucila Gibaja), (Buenos Aires: Editorial Schapire S.R.L., 1965), pág. 14.

7. Ídem., páginas 114 y siguientes.

8. André Felipe Palacio, "La comprensión clásica del suicidio. De Émile Durkheim a nuestros días\#, Andrés Felipe Palacio, Revista Affectio Societatis Vol. 7, N 12, (junio 2010): 4. http://antares.udea.edu.co/psicoan/affectio12.html 


\section{LA EUTANASIA}

La Organización Mundial de la Salud (OMS) define la Eutanasia como "aquella acción del médico que provoca deliberadamente la muerte del paciente".

El diccionario de la Real Academia de la Lengua define a la eutanasia como: "Intervención deliberada para poner fin a la vida de un paciente sin perspectiva de cura" y como segunda acepción: "Muerte sin sufrimiento físico". A la vez que ésta palabra deviene del griego euthanasía que significa "muerte dulce".

Detalla la OMS a la vez, que la Eutanasia puede realizarse de dos maneras:

a) De forma Directa, llamada también activa o voluntaria, viene a ser la acción por medio de la cual se administra directamente al enfermo una inyección letal, a través de la cual se le suministra al enfermo una muerte indolora, que unas veces puede ser solicitada por petición del paciente o también se presenta cuando el paciente no está en la capacidad de dar su consentimiento

b) De forma Indirecta, llamada también pasiva, definida como la acción por la cual se deja de suministrar al enfermo el soporte básico para su supervivencia.

Un estudio elaborado por la Psicóloga María Pilar Lampert Grassi ${ }^{9}$ para la Comisión de Salud del Congreso Nacional de Chile desarrolla una serie de categorías sobre la eutanasia:

1. Eutanasia Directa: Consiste en adelantar la hora de la muerte en caso de una enfermedad incurable, y que admite dos posibilidades:

a. Eutanasia Activa: Es aquella que consiste en efectuar las acciones específicas para provocar la muerte del paciente. La muerte de éste resulta, en consecuencia, de una acción directa (como pudiera ser la administración de dosis mortales de estupefacientes o de una substancia letal) y efectiva de un tercero; generalmente un médico.

b. Eutanasia Pasiva: En este caso la muerte resulta como consecuencia de la interrupción de intervenciones, cuyo objetivo sea simplemente ofrecer a la vida su soporte indispensable.

Ambas categorías, activa y pasiva, pueden, a su vez, ser voluntaria e involuntaria:

c. Eutanasia Voluntaria: Es aquella que se produce cuando el paciente competente (racionalmente hablando), después de discernir y evaluar la información sobre su estado de salud, solicita la eutanasia.

9. Psicóloga de la Universidad de Valparaíso, y Máster in Arts in Womwns Studies, Lancaster University, Reino Unido, E-mail: mlampert@bcn.cl. 
d. Eutanasia No-voluntaria: Se plantea cuando no se conoce, ni se puede conocer, si el paciente desea morir. La expresión se suele utilizar en los casos de pacientes en estado vegetativo que, mientras pudieron, no anticiparon directrices sobre sus preferencias de tratamiento. Debido a la situación, la decisión debe ser tomada por un tercero competente.

2. Eutanasia Indirecta: Se denomina de este modo la eutanasia cuya intención básica no es acortar la vida sino aliviar el sufrimiento. Consiste en procedimientos terapéuticos que tienen como efecto secundario la muerte, por ejemplo: la sobredosis de morfina para calmar los dolores, cuyo efecto secundario, provocaría como se sabe, una abreviación de la vida.

Concluye este estudio señalando que existen además, otras figuras jurídicas similares a la eutanasia, como es el Suicidio Asistido, consistente en proporcionar a una persona, en forma intencionada y con su autorización, los medios o procedimientos para suicidarse. ${ }^{10}$

En la actualidad hay países que cuentan con legislación de eutanasia activa o suicidio asistido como son Holanda (Reino de los Países Bajos) y Bélgica.

Un informe elaborado por la periodista Isabel Ferrer para el diario español El País en junio de 2021 sobre la eutanasia en Holanda señala que: Los Países Bajos aprobó en 2001 la primera ley de eutanasia del mundo como la culminación de un proceso madurado desde 1950, cuando se oyeron las primeras voces favorables a la práctica. La norma ha ido evolucionando con la sociedad. En 2020 hubo casi 7,000 casos, un $4,1 \%$ del total de las muertes del país. ${ }^{11}$

Señala Ferrer que entre 1970 y 2001, hubo un goteo de casos en los tribunales saldados con la absolución de los facultativos implicados, que alegaron haber actuado ante una "situación de emergencia". Presentada por la entonces Ministra de Sanidad Els Borst, médica ella misma, la norma entró en vigor en 2002, pero la eutanasia sigue considerándose una práctica extraordinaria en lugar de un derecho.

En lo que respecta a Bélgica, la cadena alemana de noticia D W (Deutsche Welle) Made for minds, destaca a este país como la pionera en la eutanasia, desde 2002, Bélgica otorga a enfermos terminales el derecho a la eutanasia. La ley de eutanasia de este país es considerada una de las más liberales del mundo. Según las estadísticas de la Comisión de Evaluación de la Eutanasia, el 67 \% de los pacientes estaba enfermo de cáncer terminal, el 19\% sufría de numerosas enfermedades (polipatología) y sólo el 3\% padecía de enfermedades psiquiátricas. Además, 3/4 partes de los pacientes tenían entre 60 y 90 años. El aumento en el número de casos en este país es notorio: después de la legalización había sólo

10. La Eutanasia en la Legislación Nacional y Extranjera, Departamento de Estudios, Extensión y Publicaciones de la Biblioteca del Congreso Nacional de Chile, 15 de mayo de 2018. Este estudio fue elaborado por la Psicóloga María Pilar Lampert Grassi para la Comisión de Salud del Congreso Chileno

11. La ley holandesa de eutanasia suma un respaldo del 87\% al cumplir 20 años, El País, El periódico global, España, Isabel Ferrer, 23 de junio de 2021. 
unos cientos al año. Actualmente hay más de 2,300 casos oficialmente registrados y la tendencia va en aumento. $^{12}$

En nuestro continente tenemos el antecedente de Colombia donde se despenalizó la eutanasia desde el año 1997 para algunos casos, bajo la sentencia C-239, en la cual se demandó la inconstitucionalidad del homicidio pietístico y la Corte decretó la constitucionalidad del homicidio por piedad siempre que se presenten tres (3) presupuestos: ${ }^{13}$

1. El sujeto pasivo debe ser mayor de edad y padecer una enfermedad terminal.

2. El sujeto activo que realiza la acción u omisión tendiente a acabar con los dolores del paciente, en todos los casos debe ser médico.

3. Debe producirse por petición expresa, reiterada e informada de los pacientes.

En el caso del Perú conforme lo señalamos en la introducción del presente trabajo, el caso de la Señora Ana Milagros Estrada Ugarte deviene en la tendencia a reconocer a la eutanasia como un derecho de poder decidir en que momento dar fin a su vida, como se sustento en su demanda, cuando los intolerables dolores de la enfermedad que padece y a las condiciones de deterioro de su salud que derivan de esta, prolongar su existencia sea incompatible con su dignidad.

Es así que el Juez del $11^{\circ}$ Juzgado Constitucional de Lima declaró fundada en parte la demanda disponiendo inaplicable el art. $112^{\circ}$ del Código Penal vigente (delito de homicidio piadoso) y disponiendo que los demandados respeten la decisión de la demandante de poner fin a su vida a través del procedimiento técnico de la eutanasia.

\section{EL CONTRATO DE SEGURO}

En nuestro ordenamiento legal y antes de la entrada en vigencia de la ley 29946, Ley del Contrato de Seguro, se regulaba el mismo en la sección octava del Libro Segundo del Código de Comercio de $1902^{14}$ (artículos $375^{\circ}$ al $429^{\circ}$ ), en ella se precisaba el carácter mercantil del contrato de seguro, sus causales de nulidad, la formalidad del mismo que establecía que se consignará por escrito, el contenido de la póliza entre otros, regulaba sólo tres tipos de seguros: contra incendios, de transporte terrestre y seguro sobre la vida; sobre éste último debemos precisar que su articulado comprendido en el $411^{\circ}$ al $422^{\circ}$ prácticamente deriva su contenido a lo establecido en la póliza respectiva refiriéndose sobre gene-

12. "Bélgica: pionera en la euthanasia", D W Made for minds, cadena alemana de noticias, 26 de febrero de 2020, https://www.dw.com.

13. "La Eutanasia y su Impacto en el Contrato de Seguro de Vida en el Ámbito Colombiano", Natalia Isabel Aristizábal y Julián Quintero Rivera, trabajo de grado realizado para optar al título de Abogado, Universidad EAFIT, Facultad de Derecho, Medellín, Colombia, 2016, pág. 12. https://repository.eafit.edu.co.

14. El Código de Comercio fue promulgado el 15 de febrero de 1902 y entró en vigencia el 01 de julio del mismo año, siendo casi una copia del Código de Comercio español de 1885. 
ralidades en su regulación; finalmente, en el artículo $429^{\circ}$ referido a seguro sobre otra clase de riesgo la citada norma señala lo siguiente: "Podrá ser asimismo objeto de seguro mercantil, cualquiera otra clase de riesgos que provengan de casos fortuitos o accidentes naturales; y los pactos que se consignen deberán cumplirse, siempre que sean lícitos y estén conformes con las prescripciones del Título de esta Sección”.

Con una vigencia de más de cien años y no obstante que la Ley N²6702, Ley General del Sistema Financiero y del Sistema de Seguros y Orgánica de la Superintendencia de Banca y Seguros promulgada en Diciembre de 1996 trató de abarcar algunos aspectos del contrato de seguro se requería una ley sobre la materia por lo que, casi a fines del año 2012 y luego de cierto batallar en el Congreso de la República se aprobó la Ley N²9946, Ley del Contrato de Seguro promulgada por el Presidente Ollanta Humala Tasso el 26 de Noviembre de dicho año, la misma que de acuerdo a la Novena Disposición Complementaria, entraría en vigencia a los 180 días desde su publicación, por lo que efectivamente rige desde el 27 de Mayo de 2013.

Esta Ley, como ya lo señalé en una publicación anterior ${ }^{15}$ consta de un primer título referido a disposiciones generales, el mismo que se compone de cuatro artículos, y dos Títulos más, uno sobre el contrato de seguro y el otro sobre el contrato de reaseguro. Ambos con 139 artículos en total y trece disposiciones entre complementarias, finales y modificatorias. Es a partir del tratamiento del seguro de personas (Título II, Capítulo III, artículo $115^{\circ}$ y siguientes) de dicha norma que el legislador comprende dentro de los mismos el seguro de vida, en cuyo artículo 125 se refiere al suicidio, materia en parte, de este trabajo.

\section{EL RIESGO}

Antes de apreciar si estas dos figuras, el suicidio y la eutanasia, son pasibles de cobertura en el seguro, debo citar un elemento esencial sobre el cual se desarrolla la actividad aseguradora: el riesgo.

Conforme al Diccionario MAPFRE de Seguros, Riesgo: en la terminología aseguradora, se emplea este concepto para expresar indistintamente dos ideas diferentes: De un lado, riesgo como objeto asegurado; de otro, riesgo como posible ocurrencia por azar de un acontecimiento que produce una necesidad económica y cuya aparición real o existencia se previene y garantiza en la póliza y obliga al asegurador a efectuar la prestación, normalmente indemnización, que le corresponde. ${ }^{16}$

15. Rolando Torres Gamero, "El contrato de seguro en el Perú, Comentarios a la Ley № 29946”, Revista Lex N 11, Facultad de Derecho y Ciencia Política de la Universidad Alas Peruanas, Año XI, (2013): pág. 152.

16. Julio Castelo Matrán, Antonio Guardiola Lozano, Diccionario MAPFRE de Seguros, (Madrid, España: Editorial MAPFRE S.A., $3^{\circ}$ Edición, noviembre 1992), pág. 333. 
Los caracteres esenciales del riesgo son los siguientes:

1. Incierto o aleatorio: Sobre el riesgo ha de haber una relativa incertidumbre, pues el conocimiento de su existencia real haría desaparecer la aleatoriedad, principio básico del seguro.

2. Posible: Ha de existir la posibilidad de riesgo, es decir, el siniestro cuyo acaecimiento se protege con la póliza debe "poder suceder". Tal posibilidad o probabilidad tiene dos limitaciones extremas: de un lado, la frecuencia, de otro, la imposibilidad.

3. Concreto: El riesgo ha de ser analizado y valorado por la aseguradora en dos aspectos: cualitativo y cuantitativo, antes de proceder a asumirlo. Sólo de esa forma la entidad podrá decidir sobre la conveniencia o no de su aceptación y, en caso afirmativo, fijar la prima adecuada.

4. Lícito: El riesgo que se asegure no ha de ir, según se establece en la legislación de todos los países, contra las reglas morales o de orden público, ni en perjuicio de terceros, pues de ser así, la póliza que lo protegiese sería nula automáticamente. Se configuran dos excepciones: el suicidio: circunstancia que lesiona el principio de orden público; y la responsabilidad civil: donde puede garantizarse los daños causados a terceros cometidos por imprudencia (aspecto legalmente sancionado por el ordenamiento penal de cualquier país).

5. Fortuito: El riesgo debe provenir de un acto o acontecimiento ajeno a la voluntad humana de producirlo.

6. Contenido económico: La realización del riesgo ha de producir una necesidad económica que se satisface con la indemnización correspondiente.

Siendo estos caracteres esenciales fundamentales para configurar el riesgo, ambas figuras materia de este trabajo afectarían los mismos en la medida que implica un accionar del propio asegurado para determinar el evento que daría fin a su propia vida, ya sea por acto propio o asistido por terceros.

\section{VI. ¿TIENE COBERTURA EL SUICIDIO?}

En el seguro de vida, señala el destacado Maestro Antígono Donati (1910-2002), el alea contractual, es decir, la relación entre la importancia económica de las prestaciones de las partes, depende de la duración de la vida humana; éste depende, a su vez, del evento de la muerte o de que no ocurra en un momento determinado (sobrevivencia). ${ }^{17}$

Refiriéndose al suicidio, el profesor de Derecho de Seguros de la Universidad de Roma señala que está considerada dentro de las delimitaciones causales subjetivas del riesgo, al respecto indica que el antiguo Código de Comercio Italiano excluía de la cobertura el suicidio voluntario y lo mismo hacían las

17. Antígono Donati, Los Seguros Privados, Manual de Derecho, (España, Barcelona: Librería Bosch ,1960), pág. 458 y sgtes. 
pólizas, adoptando expresiones varias ("suicidio", "suicidio voluntario", "suicidio consciente", "suicidio premeditado"). El concepto mismo de suicidio, así objetivado, dio lugar a numerosas cuestiones, y los aseguradores, para reducirlo y promover el espíritu de previsión, terminaron por comprender también el riesgo de suicidio, siempre que ocurra cuando el contrato ha permanecido ininterrumpidamente en vigor durante un cierto tiempo.

Concluye Donati indicando que, para favorecer el espíritu de previsión y en consideración al instinto vital, que constituye la contrapartida físicopsíquica del acto, el Código (refiriéndose al artículo $1927^{\circ}$ del Código Civil Italiano de 1942), salvo convención contraria, declara cubierto el riesgo de la muerte a causa de suicidio cuando han transcurrido dos años y las pólizas pueden abreviarlo o prolongar el término, desde la celebración del contrato o de su reactivación después de su suspensión. ${ }^{18}$

Isaac Halperín (1908-1975), profesor de la Universidad de Buenos Aires, ${ }^{19}$ comentando el artículo $135^{\circ}$ de la Ley 17.418, Ley de Seguros de Argentina de 1967, señala que por suicidio debe entenderse la muerte dada a sí mismo voluntariamente. El requisito de la voluntariedad está expreso en la ley y es inherente al suicidio, ya que el cometido en estado de inconciencia o estado de perturbación o enfermedad mental es un caso fortuito. ${ }^{20}$

$\mathrm{Al}$ respecto y siguiendo el análisis de la norma de seguros argentina, el maestro Rubén S. Stiglitz señala que el elemento voluntario tiene fuente normativa ya que lo que libera al asegurador es el "suicidio voluntario". Y así ha sido recogido por la Ley de Seguros en el art. $135^{\circ}$... Y ello es así, al punto que si el suicidio no es voluntario, es decir, cuando el asegurado no ha provocado intencionalmente el siniestro, subsiste la obligación asumida por el asegurador. ${ }^{21}$

Stiglitz precisa que el requisito de la voluntariedad excluye la hipótesis del suicidio de quien se halla con sus facultades mentales alteradas, pues en ese caso la demencia opera como evento fortuito e incierto $y$, por tanto, asegurable. ${ }^{22}$

En sintesis, afirma Stiglitz, cuando el suicidio es la resultante de un estado patológico (cita entre otros, el sonambulismo, el hipnotismo, la ebriedad alcohólica, la ingestión o aspiración de alcaloides como el opio, cocaina, morfina o similares) se equipará al caso fortuito y, por tanto, es garantizado por la relación aseguradora.

18. Antígono Donati, ídem., pág. 460.

19. Ossa G., J. Efrén, Teoría General del Seguro, El Contrato, (Bogotá, Colombia: Editorial Temis Librería 1984), pág. 91. 20. Isaac Halperín, Seguros, Exposición crítica de las leyes 17.418 y 20.091, (Buenos Aires: Ediciones Depalma, 1986), 873.

21. Rubén S. Stiglitz, “Temas de Derecho de Seguros” Colección Internacional N²3, Colombia, Bogotá: Pontificia Universidad Javeriana y Grupo Editorial Ibañez.(junio, 2010): 219.

22. Ídem, pág. 220. 
Para el Maestro colombiano Efrén Ossa (1919-1993), la asegurabilidad del suicidio viene a ser un tema particularmente espinoso. Si es o no un hecho intencional, imputable a la libre voluntad del hombre. Y si, caso de serlo, puede encajar dentro del campo de acción del seguro. No se trata de agotar, pero ni siquiera de ensayar un estudio científico de tan intrincado problema. A veces, en este como en otros casos, hay que sacrificar el rigor de la lógica jurídica en aras de la conveniencia social.

El Diccionario MAPFRE de Seguros señala que el suicidio es el acto por el que una persona da fin, voluntariamente, a su propia vida. El suicidio del asegurado - en el seguro de vida - está cubierto por la póliza cuando ocurra después del primer año de vigencia del seguro"23.

En nuestro país, la Ley 29946, Ley del Contrato de Seguro, establece en el artículo $125^{\circ}$ correspondiente al capítulo de Seguro de Vida que: "El suicidio consciente y voluntario de la persona cuya vida se asegura, libera al asegurador, salvo que el contrato haya estado en vigencia ininterrumpidamente por dos (2) años".

$\mathrm{Al}$ respecto el legislador nacional ha seguido la tendencia sobre la materia ya plasmada en normas anteriores tanto europeas como latinoamericanas siendo el caso del Ecuador el más reciente con su Código de Comercio de Mayo de 2019. ${ }^{24}$

Al comparar dichas legislaciones podemos apreciar que, como lo acotaba el Maestro Donati, los aseguradores, para reducir y promover el espíritu de previsión, terminaron por comprender el riesgo de suicidio, siempre que ocurra cuando el contrato ha permanecido ininterrumpidamente en vigor durante un cierto tiempo. Es por esto que apreciamos como hay legislaciones que establecen un período de un año, una mayoría de dos años y en el caso de la norma argentina, de tres años.

\footnotetext{
23. Ídem., pág. 376.

24. - Código Civil Italiano de 1942, artículo 1927: Suicidio del asegurado. - En caso de suicidio del asegurado, ocurrido antes de que hayan transcurrido dos años desde la estipulación del contrato, el asegurador no está obligado al pago de las sumas aseguradas, salvo pacto en contrario.

- Ley de Contrato de Seguro de España, Ley 50/1980, artículo 93: Salvo pacto en contrario, el riesgo de suicidio del asegurado quedará cubierto a partir del transcurso de un año del momento de la conclusión del contrato. A estos efectos se entiende por suicidio la muerte causada consciente y voluntariamente por el propio asegurado.

- Código de Comercio de la República de Chile, 1866, artículo 598: Provocación del siniestro y suicidio. - ... Salvo pacto en contrario, el riesgo de suicidio del asegurado sólo quedará cubierto a partir de dos años de la celebración del contrato, o de haber estado vigente el seguro por igual plazo en virtud de sucesivas renovaciones.

- Ley sobre el Contrato de Seguro de México, 31 de agosto de 1935, artículo 197º: La empresa aseguradora estará obligada, aún en caso de suicidio del asegurado, cualquiera que sea el estado mental del suicida o el móvil del suicidio, si se verifica después de dos años de la celebración del contrato. Si el suicidio ocurre antes de los dos años, la empresa reembolsará únicamente la reserva matemática.

- Ley de Seguros Argentina, Ley 17.418, 30 de agosto de 1967, artículo 135: Suicidio. - El suicidio voluntario de la persona cuya vida se asegura, libera al asegurador, salvo que el contrato haya estado en vigor ininterrumpidamente por tres años.

- Código de Comercio de Ecuador, 29 de mayo de 2019, artículo $78^{\circ}$ : En los seguros de vida contra el riesgo de muerte, sólo puede excluirse el suicidio voluntario o involuntario del asegurado ocurrido durante los dos primeros años de vigencia del contrato.
} 
En el caso de la norma de seguros mexicana existe la singularidad referida al período de carencia sobre el cual no estaría cubierto el suicidio antes de los dos años de vigencia del contrato, disponiendo el reembolso por parte de la aseguradora únicamente de la reserva matemática. Al respecto el Dr. Raúl Cervantes Ahumada, profesor de la Universidad Nacional Autónoma de México señala que este criterio se basa en un criterio fundamentalmente técnico precisando además a que se denomina reserva matemática. $^{25}$

Pero lo que aún es materia de discusión es la referida a la intencionalidad del suicidio, sobre este tema citamos al Decano de la Facultad de Derecho de Comillas ICADE, España, Abel Veiga Copo, quien inicialmente se pregunta ¿sería admisible de algún modo que el suicidio del asegurado fuere considerado como una causa de nulidad del contrato de seguro?, ‘acaso el suicida que consciente y voluntariamente se quita la vida tiene, además, la intención, o la tuvo premeditamente en el momento perfectivo del contrato de seguro, de defraudar o engañar a la aseguradora?, ¿̇e contrata con ese único motivo la cobertura de muerte? No lo creemos. Por mucho que en el pasado ya se hubieren dado supuestos de tentativas de suicidio por el propio asegurado, todas ellas frustradas. En cierto sentido queda la cuestión relegada a una decisión política comercial de la propia entidad aseguradora quien puede incluir o excluir la autólisis, o provocación de la propia muerte. ${ }^{26}$

\section{VII. ¿TIENE COBERTURA LA EUTANASIA?}

Al respecto el Maestro español Francisco Javier Tirado Suárez, profesor de Derecho Mercantil de la Universidad Complutense de Madrid y destacado especialista en Derecho de Seguros desarrolla una tesis en base al siguiente fundamento: "En nuestro Derecho no existe, al estar prohibida la eutanasia en cualquiera de sus formas, un precepto análogo al artículo 15 de la ley belga, que establece que la persona fallecida a consecuencia de una eutanasia realizada con respecto de las condiciones previstas por la presente ley se le reputará fallecida de forma natural por lo que respecta a la ejecución de cualesquiera contratos en los que fuere parte y, en particular, de los contratos de seguro. ${ }^{27}$

25. Raúl, Cervantes Ahumada, Derecho Mercantil, Primer Curso, Editorial Porrúa S.A., México, año 2000, pág. 592: "Como intermediaria en el proceso de distribución de las consecuencias económicas de los riesgos, la aseguradora forma un fondo de primas (las que han sido calculadas actuarialmente) y con dicho fondo resarce al beneficiario que ha sufrido el riesgo.

En el cálculo de las primas de un seguro de vida intervienen cálculos de probabilidades, tablas de mortalidad y otros datos técnicos. Conforme a los resultados de los cálculos actuariales, se fija una prima que deberá pagarse por cada período (generalmente anual) del contrato.

Desde el punto de vista técnico la prima puede dividirse en dos porciones: la que corresponde a la aportación al fondo de indemnizaciones y la que constituye una reserva adicional, para eventualidades. Naturalmente, las primas de los primeros años contienen una reserva mayor de las posteriores y tal reserva va disminuyendo cada año, en que, por transcurso del tiempo, se avecina el siniestro consistente en la muerte del asegurado. Como los citados elementos se calculan matemáticamente, la porción de la prima que es constitutiva de reserva se le ha llamado, técnicamente, reserva matemática. Las normas técnicas para su cálculo deberán figurar en las pólizas.

26. Abel Veiga Copo, “Suicidio y Aseguramiento", Revista de Responsabilidad Civil y Seguro, España, Año 56, N² 2, (febrero 2020): pág. 15. www.inese.es.

27. Eutanasia y seguro de personas, Francisco Javier tirado Suárez, profesor titular de Derecho Mercantil, Universidad Complutense de Madrid, https://eprints.ucm.es. 
Ahora bien, señala el Maestro Tirado Suárez, el fallecimiento del asegurado y la percepción de la suma asegurada por terceros, que, lo más probable, carezcan de toda relación con el hecho y circunstancias del suicidio del asegurado, ha hecho que el artículo 93 de la Ley de Contrato de Seguro (de España) establezca un régimen supletorio a la voluntad contractual, imponiendo que el riesgo de suicidio del asegurado quede cubierto, a partir del transcurso de un año del momento de la conclusión del contrato...Ahora bien, surge la cuestión de si la eutanasia se puede considerar un suicidio, cuando la misma no tenga carácter voluntario, hipótesis posible, a la vista de la definición de la RAE, y dado que el propio art. $93^{\circ}$ de la Ley de Contrato de Seguro fija un concepto legal de suicidio como "la muerte causada consciente y voluntariamente por el propio asegurado", se puede considerar que si no existe consentimiento expreso y específico para el acto de la eutanasia por parte del asegurado, el mismo no se puede configurar como un suicidio a los efectos del artículo $93^{\circ}$ antes citado, ya que la hipótesis sería análoga al homicidio o cualquiera de sus variantes cualificadas, por lo que existiría cobertura aseguradora en todo caso (el resaltado es nuestro).

Bajo este criterio y la que la jurisprudencia comparada ha venido estableciendo en el tiempo nos lleva a considerar que el caso de doña Ana Milagros Estrada Ugarte viene a ser un paso importante para que nuestros legisladores lo tomen en cuenta como ocurrió en Enero de 2021 por medio del Proyecto de Ley N ${ }^{\circ}$ 6976/2020-CR presentado por el congresista Gino Costa Santolalla del Partido Morado el mismo que plantea el regular el derecho que le asiste al o la paciente de pedir ayuda médica para poner fin a su vida en los supuestos especificados en dicho proyecto bajo los principios de a) Vida Digna, b) Autonomía, c) Información y d) Protección a la intimidad y confidencialidad.

Pero, además, en el artículo $6^{\circ}$ del señalado proyecto referido a la muerte por eutanasia señala: "La muerte producida por eutanasia se considerará para todo efecto equivalente a una muerte natural, incluido lo relativo a los seguros de vida, por lo que el médico responsable estará exento de responsabilidad penal"

Finalmente, dicho proyecto de ley propone derogar los artículos 112 y 113 del Código Penal referidos al homicidio piadoso y a la instigación o ayuda al suicidio, respectivamente.

\section{CONCLUSIONES}

Finalmente, y, conforme a la legislación y jurisprudencia comparada, debemos precisar lo siguiente:

1.- Tanto el suicidio como la eutanasia son figuras que alteran los caracteres esenciales del riesgo en la medida que es el propio asegurado que toma la decisión de dar fin a su vida ya sea por acto propio $\mathrm{o}$ asistido por terceros.

2.- En el caso del suicidio consciente y voluntario es admitido por la actividad aseguradora luego de un período que varía según la legislación comparada, en cambio, el suicidio involuntario o inconsciente cuenta con cobertura desde el perfeccionamiento del contrato de seguro. 
3.- Con respecto a la eutanasia debemos considerar la tendencia mundial a legalizarlo como ya ocurre en los casos citados del Reino de los Países Bajos y Bélgica, así como la jurisprudencia comparada y en el caso del Perú, el precedente judicial establecido en el caso de dońa Ana Milagros Estrada Ugarte que de por sí establecería un primer paso para considerar esta figura como un derecho que pueda ser ejercido por las personas que se encuentren en una situación de padecimiento por una enfermedad incurable que denigra su condición de ser humano.

4.- Ya existe en nuestro país un Proyecto de Ley para legislar el derecho a disponer sobre la oportunidad del paciente a disponer cuando dar fin a su vida con la asistencia médica necesaria y el reconocimiento como muerte natural con derecho a acceder a su seguro de vida.

5.- Adecuar la legislación nacional a la debida implementación de la figura de la eutanasia en la medida que deje de penalizarse la muerte asistida y la ayuda al suicidio mediante una adecuada técnica legislativa que no comprometa el accionar médico de quienes intervengan en los actos que asistan al paciente en su ejercicio de decidir cuando dar fin a su vida.

\section{REFERENCIAS}

- $\quad$ Aristizábal, Natalia Isabel; Quintero Rivera Julián. "La Eutanasia y su Impacto en el Contrato de Seguro de Vida en el Ámbito Colombiano”. Tesis para optar el título de Abogado. Universidad EAFIT, Facultad de Derecho, Medellín, Colombia, 2016. https://repository.eafit.edu.co

- "Bélgica: pionera en la euthanasia", DW Made for minds, cadena alemana de noticias, 26 de febrero de 2020, https://www.dw.com

- Cervantes, Ahumada, Raúl, Derecho Mercantil, Primer Curso. México, Editorial Porrúa S.A., año 2000.

- Matrán Julio Castelo, Lozano Antonio Guardiola, Diccionario MAPFRE de Seguros. España, Madrid: Editorial MAPFRE S.A., $3^{\circ}$ Edición, noviembre 1992.

- Donati, Antígono, Los Seguros Privados, Manual de Derecho. Traducción y notas por Arturo Vidal Sola. España, Barcelona: Librería Bosch: 1960.

- Durkheim, Émile, El Suicidio, Estudios de Sociología (versión directa del francés de Lucila Gibaja). Buenos Aires: Editorial Schapire S.R.L., 1965.

- Gob.pe, Plataforma digital única del Estado Peruano, MINSA, Noticias.

- Halperin, Isaac, Seguros, Exposición Crítica de las Leyes 17.418 y 20.091. Tomo II, Segunda Edición actualizada por Juan Carlos Félix Morandi, Argentina, Buenos Aires: Depalma Editores, julio de 1986. 
- $\quad$ OPS, Boletín Setiembre 2019, mediateam@paho.org

- OPS, Boletín Setiembre 2021, mediateam@paho.org

- Ossa G., J. Efrén, Teoría General del Seguro, El Contrato. Colombia, Bogotá: Editorial Temis Librería, 1984.

- Palacio Andrés Felipe. La comprensión clásica del suicidio. De Émile Durkheim a nuestros días. http://antares.udea.edu.co/psicoan/affectio12.html

- Stiglitz, Rubén S. y Gabriel A., Derecho de Seguros, Tomo IV, Argentina Buenos Aires: La Ley S.A.E. e I., $4^{\circ}$ Edición, $1^{\circ}$ Reimpresión, 2005.

- Stiglitz, Rubén S., “Temas de Derecho de Seguros”, Colección Internacional No 23, Bogotá, Colombia: Pontificia Universidad Javeriana y Grupo Editorial Ibañez, (junio, 2010).

- Tirado Suárez, Francisco Javier. Eutanasia y seguro de personas. Universidad Complutense de Madrid. https://eprints.ucm.es.https://eprints.ucm.es

- Torres Gamero, Rolando Javier, "El contrato de seguro en el Perú, Comentarios a la Ley $\mathrm{N}^{\circ}$ 29946", Revista Lex $N^{\circ} 11$, Facultad de Derecho y Ciencia Política de la Universidad Alas Peruanas, Año XI, (2013). https://doi.org/10.21503/lex.v11i11.8

- Veiga Copo, Abel. "Suicidio y Aseguramiento”, Revista de Responsabilidad Civily Seguro, España, Año 56, N² (febrero 2020): pág. 15. www.inese.es.www.inese.es

RECIBIDO: 12/09/2021

APROBADO:15/10/2021 